\title{
CÓDIGO NUMÉRICO DO OPENFOAM PARA ESTUDO DE MODELOS DE FLUXOS BIFÁSICOS GÁS-LÍQUIDO: VALIDAÇÃOO
}

\author{
D. M. L. de PAULA ${ }^{1}$, E. MAS DE LES VALLS ${ }^{2}$, A. M. O. SIQUEIRA $^{1}$ e L. BATET ${ }^{3}$ \\ ${ }^{1}$ Universidade Federal de Viçosa, Departamento de Química \\ ${ }^{2}$ Universitat Politècnica de Catalunya, Departamento de Máquinas e Motores Térmicos \\ ${ }^{3}$ Universitat Politècnica de Catalunya, Departamento de Física e Engenharia Nuclear \\ E-mail para contato: dayanamlouzada@gmail.com
}

\begin{abstract}
RESUMO: Este artigo tem como objetivo a validação de um código numérico para o estudo de fluxos bifásicos gás-líquido. Os resultados obtidos mostram que: 1) para a reprodução numérica de regimes turbulentos por meio de modelos laminares, torna-se necessário o emprego de uma malha bastante refinada, o que muitas vezes pode ser inviável tecnicamente; 2) a aplicação de um modelo de turbulência a uma geometria 2D, tampouco é interessante, pois a turbulência pode provocar significativa dispersão numérica, amortizando os vórtices; 3) a reprodução de um modelo de turbulência em $3 D$ produz resultados satisfatórios, mesmo por meio de uma malha mais grosseira. Com base nestas observações, é possivel afirmar que o código numérico do OpenFOAM para modelo de fluxos bifásicos gás-líquido, twoPhaseEulerFoam, é viável e adequado para representar quali e quantitativamente casos reais de modelos bifásicos, inclusive o sistema "hélio-metal líquido" dos breeding blankets de HCLL da Tecnologia de Fusão Nuclear, a partir das validações efetuadas por meio de medidas experimentais.
\end{abstract}

PALAVRAS-CHAVE: Fluxo bifásico gás-líquido; OpenFOAM; Simulação numérica.

\section{INTRODUÇÃO}

Estudos estão sendo desenvolvidos a cerca dos componentes críticos da Tecnologia de Fusão Nuclear a fim de demonstrar sua viabilidade tecnológica e comercial como fonte de energia elétrica. Um destes componentes críticos em estudo é chamado de camada fértil (breeding blanket) do tipo HCLL (Helium-Cooled Lithium-Lead), cujos canais são preenchidos com metal líquido $(\mathrm{Pb}-15,7 \mathrm{Li})$.

Neste modelo de breeding blanket, o metal líquido reage com os nêutrons acelerados provenientes da fusão, produzindo o trítio, que é posteriormente extraído e utilizado como combustível do reator de fusão. Durante esta reação de geração de trítio, o subproduto hélio é produzido na mesma proporção, o que possivelmente leva à nucleação de bolhas de hélio nos canais. A provável presença dessas bolhas no sistema HCLL levaria a um sistema bifásico gás-líquido que afetaria muito a hidrodinâmica destes canais, podendo ter consequências significativas na permeação de trítio pelas paredes do canal, na eficiência térmica do sistema e no aumento da queda de pressão nos reservatórios. Dentro deste contexto, a fim de estudar 
os efeitos dessa problemática, o objetivo deste trabalho foi validar um código numérico para modelos de fluxos bifásicos, do tipo gás-líquido, a partir da reprodução de um caso experimental de coluna de bolhas obtido da literatura científica.

\section{EQUAÇÕES GERAIS}

Para a modelagem numérica do sistema bifásico, foi selecionado o solver twoPhaseEulerFoam da ferramenta aberta OpenFOAM. Este solver utiliza o modelo de discretização Euler-Euler baseado no conceito de interpenetração contínua. Trata-se de um método simples e de fácil manipulação, onde ambas as fases são tratadas matematicamente como fases contínuas. Por outro lado, para modelar a turbulência se seguiu a estratégia RANS (Reynolds Averaged Navier-Stokes), isto é, se trabalhou com as variáveis médias no tempo e se adicionou um novo termo de tensões viscosas à equação de momento.

A fração de volume (void fraction), representada por $\alpha$ nas equações governantes, é um dos parâmetros mais importantes para caracterizar os fluxos bifásicos. Para sistemas gáslíquido, este termo pode ser definido como a fração de volume ocupada pela fase gasosa ou fase dispersa.

De acordo com Yeoh e Tu (2010), para a modelagem de fluxos bifásicos, as equações de Navier-Stokes são dadas pela equação da continuidade para cada fase $\varphi$ (Equação 1) e pela equação de conservação de momento linear (Equação 2):

$$
\begin{gathered}
\frac{\partial}{\partial \mathrm{t}}\left(\alpha_{\varphi} \rho_{\varphi}\right)+\nabla \cdot\left(\alpha_{\varphi} \rho_{\varphi} U_{\varphi}\right)=0 \\
\frac{\partial}{\partial \mathrm{t}}\left(\alpha_{\varphi} \rho_{\varphi} U_{\varphi}\right)+\nabla \cdot\left(\alpha_{\varphi} \rho_{\varphi} U_{\varphi} U_{\varphi}\right)+\nabla \cdot\left(\alpha_{\varphi} \operatorname{Re}_{\varphi, \text { eff }}\right)=-\alpha_{\varphi} \nabla p+\alpha_{\varphi} \rho_{\varphi} g+M_{\varphi}
\end{gathered}
$$

Onde o termo $\mathrm{M}_{\varphi}$ da equação de momento (Equação 2) pode ser decomposto em diferentes forças referentes à contribuição de arraste (drag), à contribuição de empuxo (lift) e à contribuição de massa virtual (virtual mass):

$$
M_{\varphi}=M_{\varphi, \text { drag }}+M_{\varphi, \text { lift }}+M_{\varphi, v m}
$$

Utilizando $a$ e $b$ como sub-índices para identificar as duas fases do sistema, onde $a$ representa a fase dispersa (gás) e $b$ a fase contínua (líquido), é possível definir de forma detalhada os termos da equação anterior. Sendo assim, o termo de arraste é descrito por: 
$\mathrm{M}_{\varphi, \mathrm{drag}}=\frac{3}{4} \alpha_{a} \alpha_{b}\left(\frac{\alpha_{b} \rho_{b} \mathrm{C}_{\mathrm{D}, \mathrm{a}}}{\mathrm{d}_{\mathrm{a}}}-\frac{\alpha_{a} \rho_{a} \mathrm{C}_{\mathrm{D}, \mathrm{b}}}{\mathrm{d}_{b}}\right)\left|\mathrm{U}_{\mathrm{r}}\right| \mathrm{U}_{\mathrm{r}}$

Onde, $U r=U a-U b$ é o vetor de velocidade relativa e $C_{D, a}$ e $C_{D, b}$ são os coeficientes de arraste para cada fase. Quando o número de Reynolds assume um valor inferior a 1.000, o coeficiente de arraste é considerado como igual a 0,44. Caso contrário, quando Re é maior ou igual a 1.000, os coeficientes $\mathrm{C}_{\mathrm{D}, \mathrm{a}}$ e $\mathrm{C}_{\mathrm{D}, \mathrm{b}}$ são obtidos de acordo com as seguintes equações:

$$
C_{D, \varphi}=\frac{24}{\operatorname{Re}_{\varphi}}\left(1+0.15 \operatorname{Re}_{\varphi}{ }^{0.687}\right)
$$

Da mesma forma, o termo referente à contribuição de empuxo é descrito pela equação:

$$
\mathrm{M}_{\varphi, \text { lift }}=\alpha_{a} \alpha_{b}\left(\alpha_{b} \mathrm{C}_{\mathrm{l}, \mathrm{a}} \rho_{b}-\alpha_{a} \mathrm{C}_{\mathrm{l}, \mathrm{b}} \rho_{a}\right) \mathrm{U}_{\mathrm{r}} \times \nabla \times \mathrm{U}_{\mathrm{b}}
$$

E a força de virtual mass, que é a inércia adicionada a um sistema quando um corpo em aceleração ou desaceleração move (ou desvia) algum volume do fluido envolvente à medida que esse corpo o atravessa, é representada de acordo com a seguinte equação:

$$
\mathrm{M}_{\varphi, \mathrm{vm}}=\alpha_{\mathrm{a}} \alpha_{b} \mathrm{C}_{\mathrm{vm}} \rho_{b}\left(\frac{\mathrm{Db}}{\mathrm{Dt}} \mathrm{U}_{\mathrm{b}}-\frac{\mathrm{Da}}{\mathrm{Dt}} \mathrm{U}_{a}\right)
$$

Para adicionar o efeito de turbulência ao sistema bifásico disperso, se utiliza um modelo $\kappa-\varepsilon$ de equações para a fase contínua e se adota a viscosidade de turbulência para os cálculos da fase dispersa. Dessa maneira, a equação de energia cinética de turbulência $\left(\mathrm{k}_{\mathrm{b}}\right)$ da fase contínua é representada por:

$\frac{\partial\left(\alpha_{b} \mathbf{k}_{b}\right)}{\partial \mathrm{t}}+\nabla \cdot\left(\alpha_{b} \mathrm{U}_{b} \mathbf{k}_{b}\right)-\nabla^{2}\left(\sigma_{\mathrm{k} b, \mathrm{eff}} \mathbf{k}_{b}\right)=\alpha_{b} \mathrm{G}-\alpha_{b} \varepsilon_{b}$

Enquanto que a taxa de dissipação de turbulência $\left(\varepsilon_{\mathrm{b}}\right)$ é calculada com a equação de transporte: 


$$
\frac{\partial\left(\alpha_{b} \varepsilon_{b}\right)}{\partial \mathrm{t}}+\nabla \cdot\left(\alpha_{b} \mathrm{U} \varepsilon_{b}\right)-\nabla^{2}\left(\sigma_{\varepsilon b, \mathrm{eff}} \varepsilon_{b}\right)=\mathrm{C}_{1} \alpha_{b} \mathrm{G} \frac{\varepsilon_{b}}{\kappa_{b}}-\mathrm{C}_{2} \alpha_{b} \frac{\varepsilon_{b}{ }^{2}}{\kappa_{b}}
$$

\section{CONDIÇÕES DO EXPERIMENTO E DA SIMULAÇÃO}

A fim de comprovar que o twoPhaseEulerFoam pode ser aplicado para a modelagem numérica de fluxos bifásicos em geral, o experimento de Becker et al. (1994) foi selecionado e utilizado para ser reproduzido com o modelo apresentado. Este experimento foi realizado em uma coluna de $2 \mathrm{~m}$ de altura, 0,5 $\mathrm{m}$ de comprimento e $0,08 \mathrm{~m}$ de profundidade, onde a fase contínua (água) ocupava um nível de $1,5 \mathrm{~m}$ na coluna e a fase dispersa (ar) era injetada por meio de um aerador de $0,04 \mathrm{~m}$ de diâmetro (Figura 1). Esse distribuidor de ar estava localizado na base da coluna, centrado no eixo $\mathrm{z}$ e situado a $0,15 \mathrm{~m}$ da parede esquerda no eixo $\mathrm{x}$.

Os testes foram realizados para duas vazões volumétricas diferentes para a entrada de ar: uma de $8 \mathrm{~L} / \mathrm{min}(\mathrm{w}=3,3 \mathrm{~mm} / \mathrm{s})$ e outra de $1,6 \mathrm{~L} / \mathrm{min}(\mathrm{w}=0,66 \mathrm{~mm} / \mathrm{s})$. Para a velocidade de entrada maior (vazão volumétrica maior), foi observada a formação de uma pluma de bolhas de ar ascendente rente à parede esquerda da coluna, devido à assimetria na localização do aerador. Isto provocou a geração de um vórtice dextrogiro que ocupou toda a altura da coluna. O fluxo chegou a condições praticamente estacionárias (Figura 3.b).

Em seguida, a vazão volumétrica foi reduzida para 1,6 L/min e o comportamento do fluxo bifásico foi alterado drasticamente. Com a velocidade baixa (vazão volumétrica menor) se formaram mais vórtices ao longo da coluna e a parte inferior da pluma de bolhas se manteve bastante estável e próxima da parede esquerda, enquanto a parte superior da pluma adquiriu uma forma mais serpenteante, que se movia de acordo com o movimento dos vórtices da fase líquida (Figura 4.b).
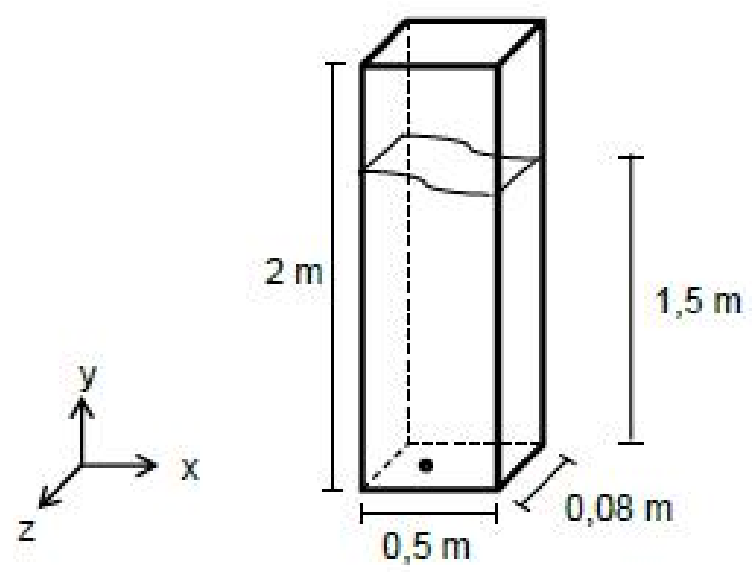

Figura 1 - Dimensões da coluna utilizada no experimento.

Baseado na coluna de Becker et al. (1994), para a simulação numérica foram utilizadas 3 malhas em 2D: M1 (50x100x1), M2 (75x150x1) e M3(100x225x1) e uma malha em 3D: M4 (28x80x4). 


\section{RESULTADOS E DISCUSSÃO}

A partir dos resultados e do comportamento do experimento de Becker et al. (1994), seja para velocidade alta ou baixa, pode-se inferir que se tratam de regimes turbulentos. Desta maneira, para realizar os testes com o twoPhaseEulerFoam foi levado em consideração que para representar bem um regime que possa ser de fluxo turbulento existem duas opções: a primeira é por meio do que se chama, neste trabalho, como um "modelo laminar", onde se utilizam as equações básicas de fluxo bifásico e uma malha suficientemente refinada; e a segunda opção é por meio de um modelo com turbulência, chamado neste trabalho de "modelo de turbulência", onde se emprega uma malha grossa e as equações básicas acopladas às equações do modelo de turbulência k- $\varepsilon$. A partir destas considerações, as simulações foram realizadas da seguinte maneira: o modelo laminar foi aplicado a 3 malhas diferentes em 2D e o modelo turbulento foi aplicado a malhas grosseiras em $2 \mathrm{D}$ e em 3D, de acordo com o esquema da Figura 2.

As geometrias em 2D foram utilizadas inicialmente, pois normalmente para colunas de pouca profundidade se supõe que o fluxo é basicamente bidimensional. Porém, com a presença do regime turbulento, a representação em 2D fica mais difícil, o que torna a geometria 3D mais interessante, pois aproxima a malha da geometria real.

Quanto aos resultados obtidos, as simulações geradas com o modelo laminar em 2D não obtiveram boa reprodutibilidade entre as malhas M1, M2 e M3, o que garante que a malha suficientemente refinada necessária para reproduzir adequadamente o sistema com o modelo laminar, tanto para a velocidade baixa como a alta, ainda estaria distante da malha M3. Como refinar ainda mais essa malha seria inviável devido ao tempo de processamento da simulação, tornou-se necessário adicionar às malhas em geometria $2 \mathrm{D}$ as equações de turbulência.

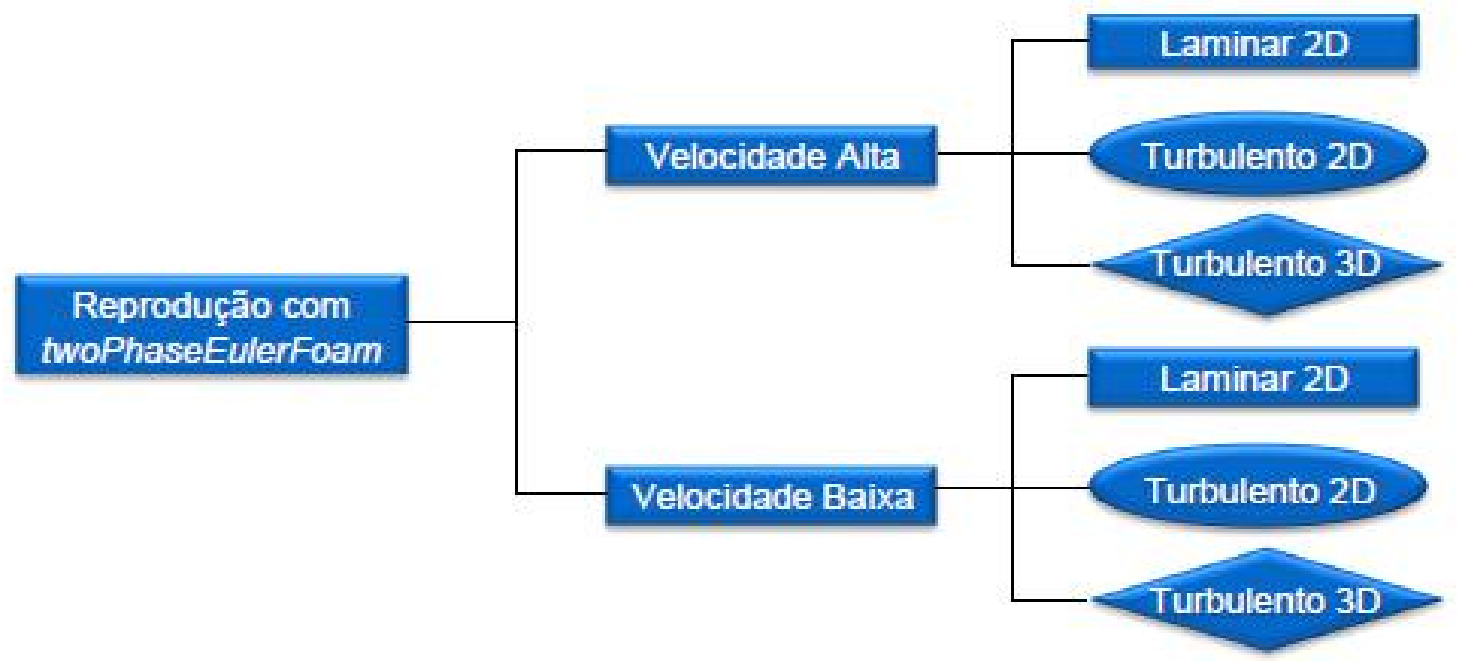

Figura 2 - Sequência de simulações realizadas. 
Dessa maneira, adicionando-se as equações do modelo de turbulência k- $\varepsilon$ e repetindo a simulação para a geometria 2D, o que se obteve foi um sistema extremamente estabilizado para as malhas M1, M2 e M3. Para ambas as velocidades, a simulação alcançou o regime estacionário dentro de poucos segundos.

Comparando este resultado com o obtido por outros autores, como Sokolichin e Eigenberger (1999) e Mudde e Simonin (1999), pôde-se concluir que as equações de turbulência sobrestimam a viscosidade efetiva da fase contínua, resultando em um sistema muito simplificado devido às limitações de profundidade e às simplificações da geometria 2D. Em contrapartida, como na geometria do tipo $3 \mathrm{D}$ não existem tais simplificações, os resultados deveriam ser mais reais e consistentes com o experimento.

Desta forma, para os últimos testes, retomou-se a ideia inicial de que um modelo mais complexo, englobando equações básicas e equações de turbulência em uma geometria mais completa, como a geometria $3 \mathrm{D}$, pode representar muito bem um experimento em regime turbulento utilizando-se apenas uma malha mais grosseira, como M4. Tendo isto em vista, testes diversos foram realizados e os resultados obtidos foram satisfatórios.

Para a velocidade alta (vazão de $8 \mathrm{~L} / \mathrm{min}$ ), as simulações convergiram para um resultado quase estacionário, obtendo comportamento e velocidades muito próximos ao obtido experimentalmente (Figura 3). A pluma de bolhas se manteve próxima da parede esquerda, promovendo a formação de um grande vórtice ao longo de toda a coluna e de um fluxo descendente de bolhas à direita.

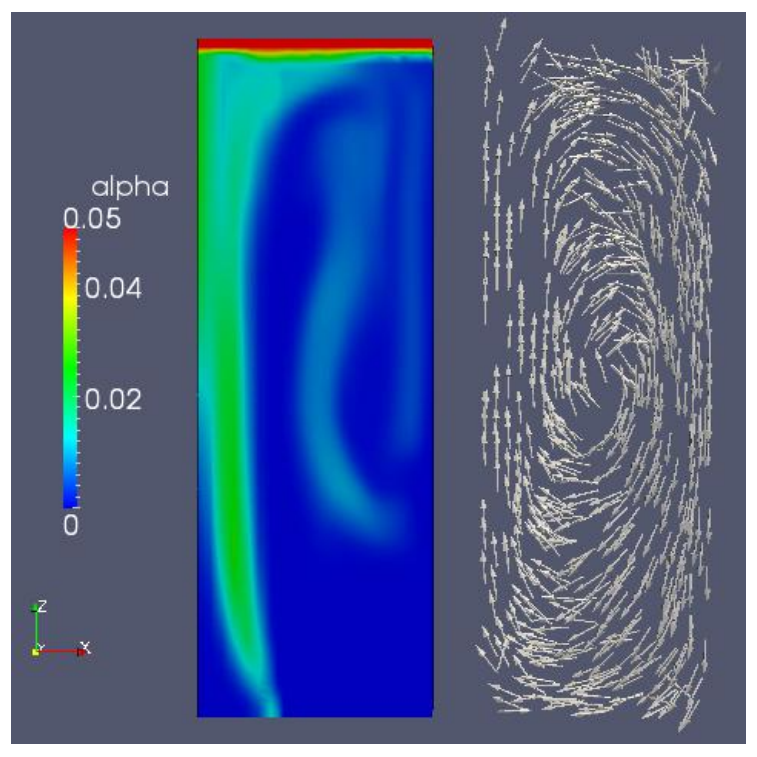

(a)
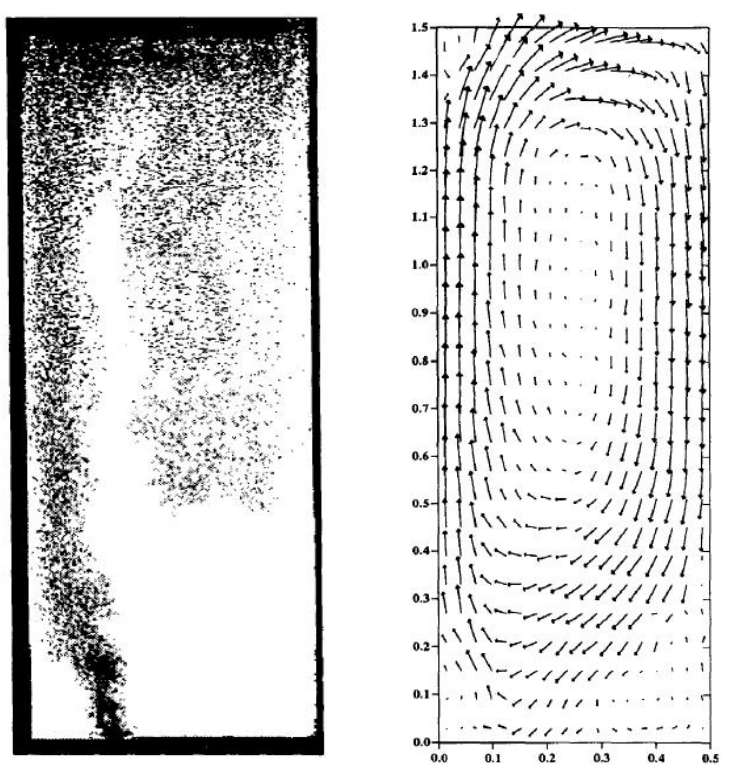

(b)

Figura 3 - Comportamento da fração de volume do ar e da velocidade do líquido:

(a) twoPhaseEulerFoam e (b) experimento. 
Igualmente, os resultados obtidos para a velocidade baixa (vazão de 1,6 L/min) foram próximos do que se obteve no caso de Becker et al. (1994). Isto é, o comportamento do fluxo foi de acordo com o que se esperava, pois a pluma obtida foi sinuosa e os vórtices formados pelo movimento da fase contínua ficaram em constante oscilação de cima para baixo e de baixo para cima (Figura 4).

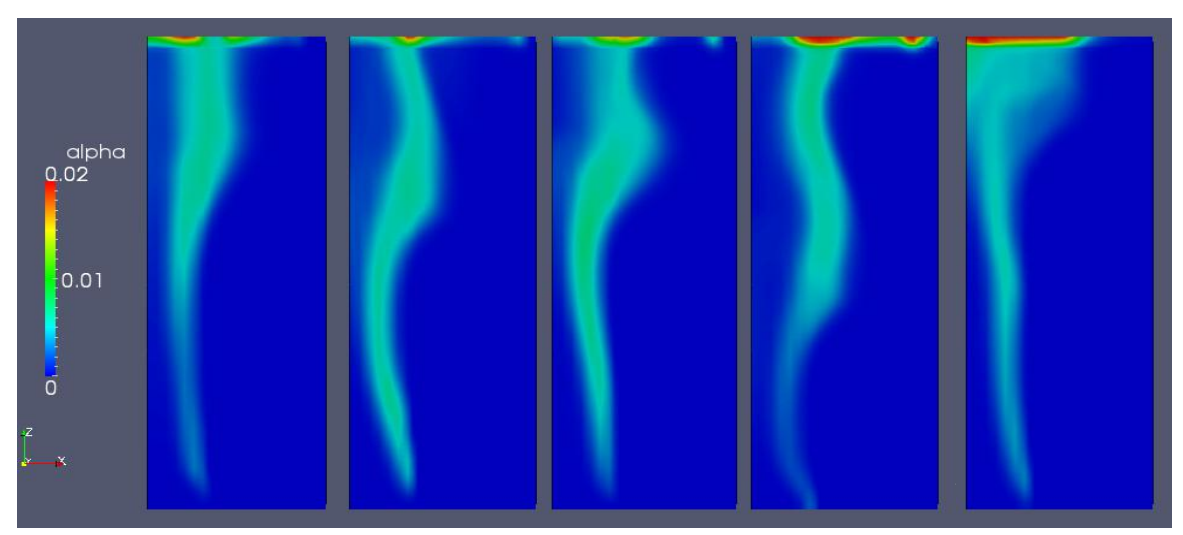

(a)
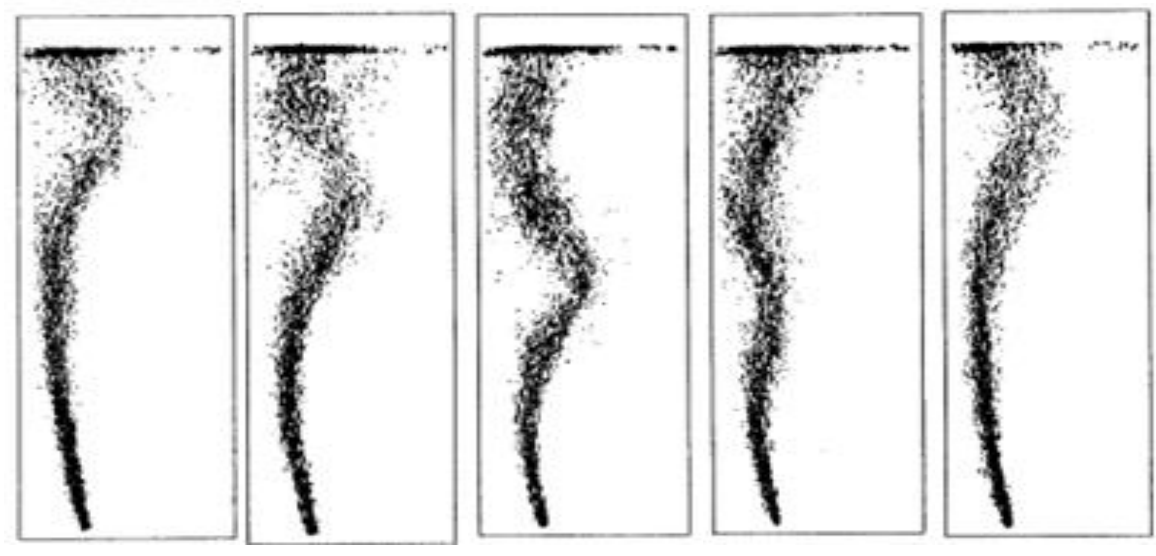

(b)

Figura 4 - Comportamento da fração de volume da fase dispersa no meio contínuo.

(a) twoPhaseEulerFoam e (b) experimento.

Além disso, ainda para a velocidade baixa, comparando os valores estimados da velocidade vertical do líquido, ao longo do tempo, com os medidos durante o experimento para dois pontos distintos da coluna (ponto A e ponto B), é possível confirmar que o comportamento da fase contínua obtido com a simulação foi similar ao do caso real (Figura 5). 

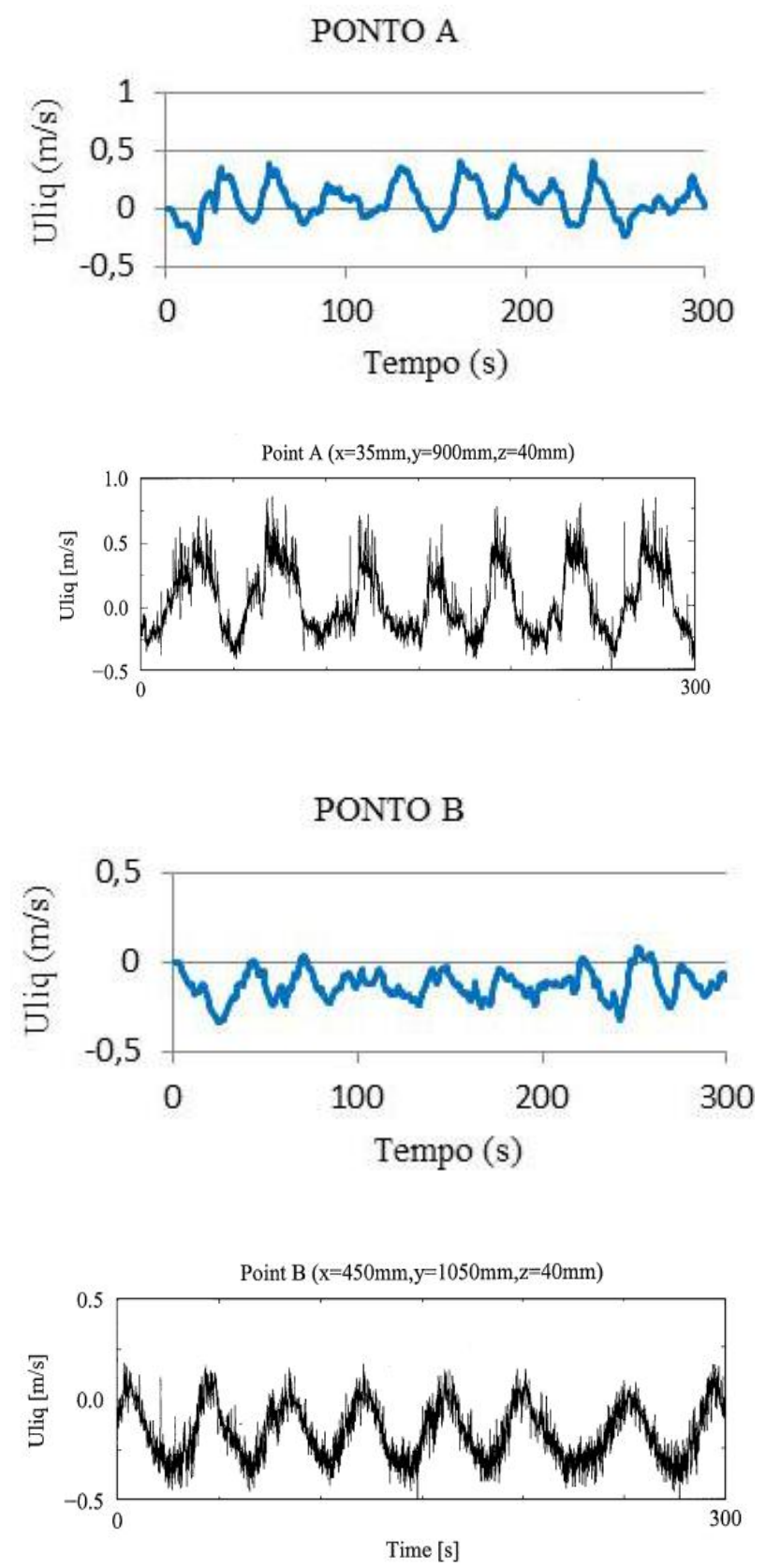

Figura 5 - Comportamento da velocidade vertical do líquido $(\mathrm{m} / \mathrm{s})$ : twoPhaseEulerFoam e experimento, nos pontos A e B. 


\section{CONCLUSÕES}

Quanto aos resultados obtidos com as simulações do código numérico do OpenFOAM, pode-se concluir que:

- Para reproduzir regimes turbulentos com modelos laminares, isto é, modelos que contenham simplesmente as equações básicas de conservação da massa e troca de momento, sem levar em consideração os parâmetros de turbulência, é necessária uma malha demasiado fina para um bom resultado. Isto pode tornar o modelo laminar inviável para alguns casos em regimes turbulentos;

- Aplicar um modelo de turbulência a uma geometria 2D, tampouco é interessante, pois a turbulência provoca uma dispersão numérica muito grande, amortizando quase todos os vórtices e movimentos dos fluidos, resultando em um sistema muito estável e estacionário;

- Por outro lado, a reprodução de um modelo de turbulência em 3D produz resultados satisfatórios e mais condizentes com casos experimentais. Dessa forma, é possível chegar a bons resultados quantitativos e qualitativos, mesmo que seja utilizando uma malha grossa;

- Além disso, testes realizados comprovaram também que para a geometria $3 \mathrm{D}$, o uso de esquemas UPWIND para cálculo de variáveis, que não sejam de turbulência, provoca muita difusão numérica. Portanto, esquemas TVD são preferíveis e produzem melhores resultados.

Sendo assim, tendo em conta essas observações, é possível afirmar que o código numérico do OpenFOAM, para modelo de fluxos bifásicos gás-líquido (twoPhaseEulerFoam), é viável para representar adequadamente casos reais de modelos bifásicos. Para trabalhos futuros, se pretende utilizar o twoPhaseEulerFoam para estudar a influência da formação de bolhas de hélio na hidrodinâmica do metal líquido dos canais do breeding blanket. Desta forma, é possível afirmar que o código numérico para modelos de fluxos bifásicos gás-líquido ( $t w o P h a s e E u l e r F o a m$ ), é viável para representar adequadamente casos reais de modelos bifásicos, inclusive para estudar a influência da formação de bolhas de hélio na hidrodinâmica do metal líquido dos canais do breeding blanket.

\section{NOMENCLATURA}

$\mathrm{C}_{\mathrm{D}} \quad$ Coef. de arraste (drag) [-]

$\mathrm{C}_{\text {lift }} \quad$ Coef. de empuxo (lift) [-]

$\mathrm{C}_{\mathrm{vm}} \quad$ Coef. de massa virtual (virtual mass) [-]

$\mathrm{C}_{\mathrm{M}} \quad$ Coef. do modelo de turbulência k- $\varepsilon$ [-]

$\mathrm{C}_{t} \quad$ Coef. de resposta de turbulência [-]

$\mathrm{C}_{1} \quad$ Const. do modelo de turbulência k- $\varepsilon$ [-]

$\mathrm{C}_{2} \quad$ Const. do modelo de turbulência k- $\varepsilon$ [-]

D Diâmetro das partículas [m] $\alpha \quad$ Fração de volume da fase dispersa [-] Fração de volume da fase contínua [-]

$\varepsilon \quad$ Taxa de dissipação turbulenta $\left[\mathrm{m}^{2} / \mathrm{s}^{3}\right]$

$\kappa \quad$ Energia cinética de turbulência $\left[\mathrm{m}^{2} / \mathrm{s}^{2}\right]$

$v \quad$ Viscosidade cinemática $\left[\mathrm{m}^{2} / \mathrm{s}\right]$

$v_{\text {eff }}$ Viscosidade cinemática efetiva $\left[\mathrm{m}^{2} / \mathrm{s}\right]$

$\rho \quad$ Densidade $\left[\mathrm{kg} / \mathrm{m}^{3}\right]$

$\tau \quad$ Tensor de tensões laminares $\left[\mathrm{N} / \mathrm{m}^{2}\right]$ 
g Aceleração da gravidade $\left[\mathrm{m} / \mathrm{s}^{2}\right]$

G Produção de energia cinética de turb. [-]

M Transf. de momento na interface $\left[\mathrm{kg} \cdot \mathrm{m}^{2} / \mathrm{s}^{2}\right]$

$\mathrm{M}_{\text {drag }}$ Momento de arraste $\left[\mathrm{kg} \cdot \mathrm{m}^{2} / \mathrm{s}^{2}\right]$

$\mathrm{M}_{\text {lift }} \quad$ Momento de empuxo $\left[\mathrm{kg} \cdot \mathrm{m}^{2} / \mathrm{s}^{2}\right]$

$\mathrm{M}_{\mathrm{vm}} \quad$ Momento de massa virtual $\left[\mathrm{kg} \cdot \mathrm{m}^{2} / \mathrm{s}^{2}\right]$

$\mathrm{P} \quad$ Pressão [Pa]

Re Tensor de tensões de Reynolds [-]

T Tempo [s]

U Vetor de velocidade $[\mathrm{m} / \mathrm{s}]$

W Velocidade superficial $[\mathrm{m} / \mathrm{s}]$

\section{REFERÊNCIAS}

$\sigma_{\mathrm{k}} \quad$ Numero de Schimidt para turb. [-]

$\varphi \quad$ Fase dispersa o contínua [-]

BECKER, S.; SOKOLICHIN, A.; EIGENBERGER, G. Gas-liquid flow in bubble columns and loop reactors: Part II. Comparison of detailed experiments and flow simulations. Chem. Eng. Science, v. 49, n. 24, p. 5747-5762, 1994.

MUDDE, R. F.; SIMONIN, O. Two- and three-dimensional simulations of a bubble plume using a two fluid model. Chem. Eng. Science, v. 54, n. 21, p. 5061-5069, 1999.

SOKOLICHIN, A.; EIGENBERGER, G. Applicability of the standard k- $\varepsilon$ turbulence model to the dynamic simulation of bubble columns: Part $I$. Detailed numerical simulations. Chem. Eng. Science, v. 54, n. 13-14, p. 2273-2284, 1999.

YEOH, G. H.; TU, J. Computational Techniques for Multi-Phase Flows. $3^{\text {rd }}$ ed. Oxford, UK: Elsevier Ltd, 2010. 


\title{
CÓDIGO NUMÉRICO OPENFOAM PARA ESTUDIO DE MODELOS DE FLUJO BIFÁSICO GAS-LÍQUIDO: VALIDACIÓN
}

\begin{abstract}
RESUMEN: En este trabajo el modelo y el solver twoPhaseEulerFoam fue validado a partir de datos experimentales y soluciones de otros autores para el estudio de flujos bifásicos gaslíquido. A partir de los resultados de la validación del modelo del OpenFOAM, es posible concluir que: 1) para reproducir regímenes turbulentos con modelos laminares, es decir simplemente con las ecuaciones básicas del modelo sin tener en cuenta la turbulencia, es necesario una malla demasiado fina para una buena solución. Lo que puede tornar el laminar inviable para algunos casos de regímenes turbulentos;2) aplicar un modelo de turbulencia a una geometría 2D, tampoco es interesante, pues la turbulencia provoca una dispersión muy grande, amortiguando todos los vórtices y movimientos de los fluidos, llevando a un sistema muy estable y estacionario; 3) por otro lado, la reproducción en $3 D$ juntamente con un modelo de turbulencia produce resultados satisfactorios comparados con el experimento. Es posible llegar a buenos resultados cuantitativos y cualitativos, mismo que sea con una malla gruesa; Además, soluciones obtenidas también comprobaron que para la geometría 3D, el uso de esquemas UPWIND para cálculo de variables que no sean de turbulencia provoca la difusión numérica, forzando el sistema al estacionario. Por lo tanto, esquemas TVD son preferibles y producen mejores resultados en $3 D$.
\end{abstract}

PALABRAS-CLAVE: Flujo bifásico gas-líquido; OpenFOAM; Simulacion numérica. 